\title{
PROPAGATION OF SECONDARY ANTIPROTONS AND COSMIC RAYS IN THE GALAXY
}

\author{
I. V. Moskalenko ${ }^{12}$, A. W. Strong ${ }^{3}$, J. F. Ormes ${ }^{1}$, and S. G. Mashnik ${ }^{4}$ \\ ${ }^{1}$ NASA/Goddard Space Flight Center, Code 661, Greenbelt, MD 20771, U.S.A. \\ ${ }^{2}$ Joint Center for Astrophysics, University of Maryland, Baltimore County, Baltimore, MD 21250, U.S.A. \\ ${ }^{3}$ MPI für extraterrestrische Physik, Postfach 1603, D-85740 Garching, Germany \\ ${ }^{4}$ Los Alamos National Laboratory, Los Alamos, NM 97545, U.S.A.
}

\begin{abstract}
Recent measurements of the cosmic ray (CR) antiproton flux have been shown to challenge existing CR propagation models. It was shown that the reacceleration models designed to match secondary to primary nuclei ratios (e.g., B/C) produce too few antiprotons. In the present paper we discuss one possibility to overcome these difficulties. Using the measured antiproton flux and $\mathrm{B} / \mathrm{C}$ ratio to fix the diffusion coefficient, we show that the spectra of primary nuclei as measured in the heliosphere may contain a fresh local "unprocessed" component at low energies perhaps associated with the Local Bubble, thus decreasing the measured secondary to primary nuclei ratio. The independent evidence for SN activity in the solar vicinity in the last few Myr supports this idea. The model reproduces antiprotons, B/C ratio, and elemental abundances up to $\mathrm{Ni}(Z \leq 28)$. Calculated isotopic distributions of $\mathrm{Be}$ and $\mathrm{B}$ are in perfect agreement with CR data. The abundances of three "radioactive clock" isotopes in $\mathrm{CR},{ }^{10} \mathrm{Be},{ }^{26} \mathrm{Al},{ }^{36} \mathrm{Cl}$, are all consistent and indicate a halo size $z_{h} \sim 4 \mathrm{kpc}$ based on the most accurate data taken by the ACE spacecraft.
\end{abstract}

\section{INTRODUCTION}

The result of our recent analysis (Moskalenko et al., 2001b, 2002), in agreement with calculations of other authors (Molnar and Simon, 2001), was that matching the secondary/primary nuclei ratio B/C using reacceleration models leads to values of the spatial diffusion coefficient apparently too large to produce the required $\bar{p}$ flux, when the propagated nucleon spectra are tuned to match the local $p$ and He flux measurements. Assuming the current heliospheric modulation models are approximately right, we have the following possibility (Moskalenko et al., 2003) to reconcile the $\mathrm{B} / \mathrm{C}$ ratio with the measured flux of secondary $\bar{p}$ 's. The spectra of primary nuclei as measured in the heliosphere may contain a fresh local "unprocessed" component at low energies thus decreasing the measured secondary to primary nuclei ratio. This component would have to be local in the sense of being specific to the solar neighbourhood, so that the well-known "Local Bubble" phenomenon is a natural candidate.

The low-density region around the Sun, filled with hot H I gas, is called the Local Bubble (hereafter LB) (e.g., Sfeir et al., 1999). The size of the region is about $200 \mathrm{pc}$, and it is likely that it was produced in a series of supernova (SN) explosions, with the last SN explosion occuring approximately 1-2 Myr ago, or $3 \mathrm{SN}$ occuring within the last 5 Myr. Most probably its progenitor was an OB star association (e.g., Ma íz-Apellániz, 2001; Berghöfer and Breitschwerdt, 2002). Knie et al. (1999) report about an excess of ${ }^{60} \mathrm{Fe}$ measured in a deep ocean core sample of ferromanganese crust suggesting a SN explosion about $5 \mathrm{Myr}$ ago at $30 \mathrm{pc}$ distance and the deposition of SN-produced iron on earth. Sonett et al. (1987) report an enhancement in the CR intensity dated about $40 \mathrm{kyr}$ ago, which is interpreted as the passage across the solar system of the shock wave from a SN exploding about $0.1 \mathrm{Myr}$ ago. It could also be that "fresh" LB contributions from continuous acceleration in the form of shock waves (Bykov and Fleishman, 1992), and/or energetic particles coming directly from SN remnants still influence the spectra and abundances of local CR. 

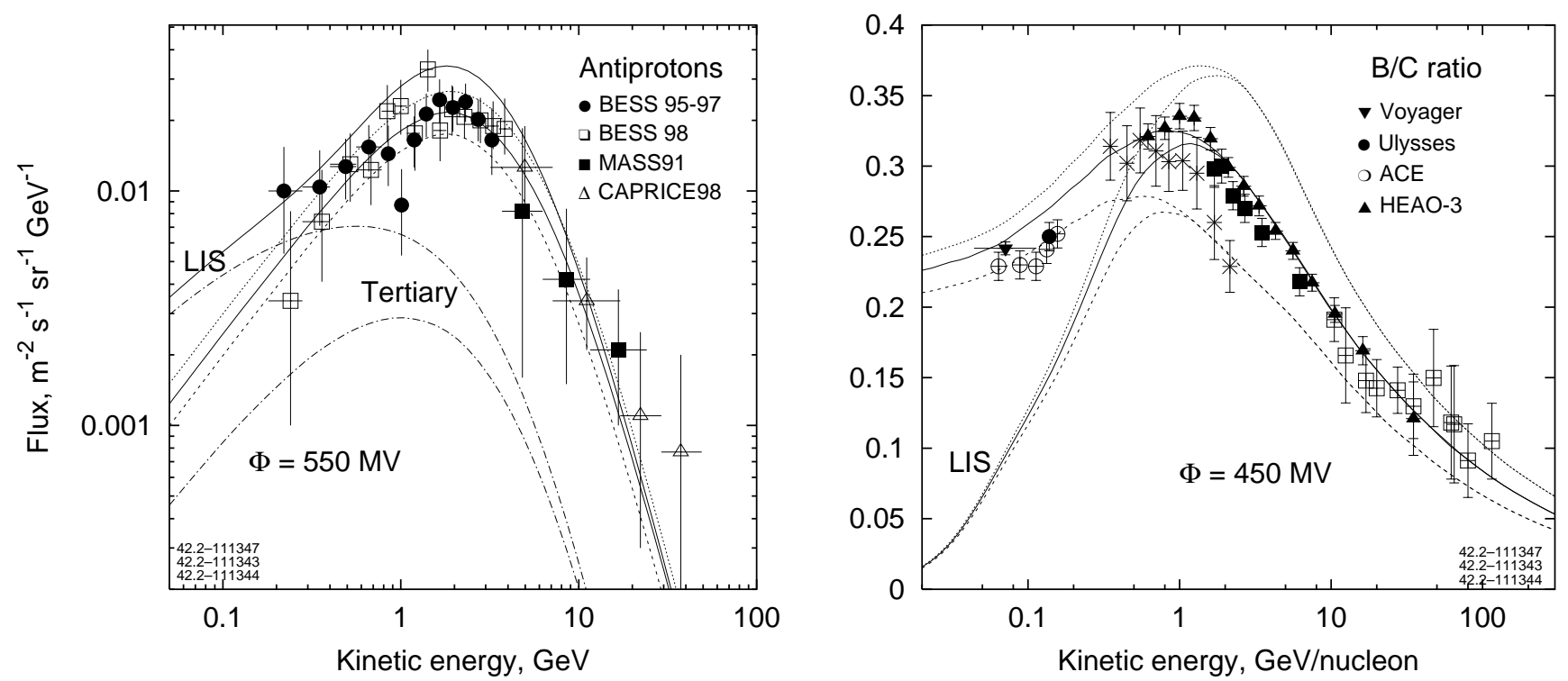

Fig. 1. Left: Calculated $\bar{p}$ flux in a model with $\delta=0.47$ and different normalization values $D_{0}\left(\times 10^{28} \mathrm{~cm} \mathrm{~s}^{-2}\right)$. Solid curves $-D_{0}=3.3$ at $\rho_{0}=3 \mathrm{GV}$, upper curve - local interstellar (LIS), lower curve - modulated ( $\Phi=550 \mathrm{MV}$ ). Dots $-D_{0}=2.6$, dashes $-D_{0}=4.3$. Data: BESS 95-97 (Orito et al., 2000), BESS 98 (Asaoka et al., 2002) MASS91 (Basini et al., 1999), CAPRICE98 (Boezio et al., 2001). Right: B/C ratio calculated with LB contribution, $E_{b}=500$ $\mathrm{MeV}$. The lines are coded as on the left panel. Lower curves - LIS, upper - modulated $(\Phi=450 \mathrm{MV})$. Data below $200 \mathrm{MeV} / \mathrm{n}$ : ACE (Davis et al., 2000), Ulysses (DuVernois et al., 1996b), Voyager (Lukasiak et al.,, 1999); high energy data: HEAO-3 (Enqelmann et al., 1990), for other references see Stephens and Streitmatter (1998).

\section{THE CALCULATION PROCEDURE}

We use the propagation model GALPROP ${ }^{1}$ as described elsewhere (Strong and Moskalenko, 1998; Moskalenko et al., 2002); for the present purpose the $2 \mathrm{D}$ cylindrically symmetrical option is sufficient. The spatial diffusion coefficient is taken as $D_{x x}=\beta D_{0}\left(\rho / \rho_{0}\right)^{\delta}$; the diffusion in momentum space and other details can be found in our earlier papers. The nucleon injection spectrum of the Galactic component was taken as a modified power-law in rigidity (Jones et al., 2001), $d q(p) / d \rho \propto \rho^{-\gamma} / \sqrt{1+(\rho / 2)^{-2}}$, for the injected particle density. The heliospheric modulation is treated using the force-field approximation. The LB spectrum is taken in the form (Bykov and Fleishman, 1992, for continous acceleration by interstellar shocks): $d f / d \rho \propto \rho^{-\eta} \exp \left(-\rho / \rho_{b}\right)$, where $\rho$ is the rigidity, and $\rho_{b}$ is the cut off rigidity parameter. In terms of kinetic energy per nucleon $E$ this can be re-written as $d f / d E=a(Z, A) \frac{A(E+m)}{Z p} \rho^{-\eta} e^{-\rho / \rho_{b}}$, where $a(Z, A)$ is the abundance of a nucleus $(Z, A), Z, A$ are the nucleus charge and atomic number, correspondingly, $m$ is the atomic mass unit, $p$ is the momentum per nucleon, $\rho_{b}=\frac{A}{Z} \sqrt{\left(E_{b}+m\right)^{2}-m^{2}}$. We show the results obtained with $\eta=1$, but the particular spectral shape of the LB component is not important as long as it decreases sharply towards high energies and is much softer than the Galactic CR spectrum.

The procedure to tune the CR elemental abundances, secondary/primary nuclei ratios, and $\bar{p}$ flux we adopted was as follows. The high energy part of $\mathrm{B} / \mathrm{C}$ ratio and $\bar{p}$ flux measurements are used to restrict the parameters of the diffusion coefficient $D_{0}$ and $\delta$, while the low energy part of $\mathrm{B} / \mathrm{C}$ ratio is used to fix a value for the reacceleration level and define the parameters of the LB component. The Galactic CR elemental source abundances are tuned using the $\mathrm{CR}$ abundances at high energies where the heliospheric modulation is weak. The LB isotopic abundances are tuned to match the low-energy data by ACE and Ulysses.

Figure 1(left) illustrates the process of fixing the normalization of the diffusion coefficient using $\bar{p}$ 's. The $\bar{p}$ flux shown is calculated with $\delta=0.47$ and different normalizations in the diffusion coefficient, $D_{0}=2.6,3.3,4.3 \times 10^{28}$ $\mathrm{cm} \mathrm{s}^{-2}$ at $\rho=3 \mathrm{GV}$ (for $\bar{p}$ 's this corresponds to kinetic energy $\sim 2 \mathrm{GeV}$ ). The injection index $\gamma$ is taken equal to 2.28 , and the Alfvén speed $v_{A}=23 \mathrm{~km} \mathrm{~s}^{-1}$. The $\bar{p}$ flux at maximum, $\sim 2 \mathrm{GeV}$, appears to be quite sensitive to the value of the diffusion coefficient and allows us to fix it at $D_{0}=(3.3 \pm 0.8) \times 10^{28} \mathrm{~cm} \mathrm{~s}^{-2}$. The exact value of $\delta$ is not critical since we compare with the $\bar{p}$ measurements at maximum. Inelastically scattered $\bar{p}$ 's, the "tertiary" component, appears to be important at low energies only in the ISM. Figure 1 (right) shows corresponding calculations of the B/C ratio. A halo height of $z_{h}=4 \mathrm{kpc}$ is used (Strong and Moskalenko, 2001; Moskalenko et al., 2001a).

\footnotetext{
${ }^{1}$ GALPROP model including software and data sets is available at http://www.gamma.mpe-garching.mpg.de/ aws/aws.html
} 
The local interstellar (LIS) $p$ and He spectra used in our $\bar{p}$ calculations are the best fits to the data as described in Moskalenko et al. (2002). Because of the measurements with large statistics (BESS - Sanuki et al. 2000, AMS - Alcaraz et al. 2000), and weak heliospheric modulation above $10 \mathrm{GeV}$, the error arising from uncertainties in the primary spectra is only $\sim 5 \%$. We note that as in the case of other nuclei, there should be an LB contribution to $p$ and He spectra. The Galactic injection spectra of $p$ 's and He should thus be significantly flatter below several $\mathrm{GeV}$ to match the data points at low energies. This does not influence the $\bar{p}$ production because (i) the LB does not produce a significant amount of secondaries, and (ii) the $\bar{p}$ threshold production energy is high, $\sim 10 \mathrm{GeV}$.

Further tuning can be done using the high energy part of the $\mathrm{B} / \mathrm{C}$ ratio, which is not influenced by heliospheric modulation and supposedly contains only a Galactic component of CR. Index $\delta \sim 0.47$ is chosen as giving the best match. A pure Kolmogorov spectrum, $\delta=1 / 3$, thus seems to be excluded by $\bar{p}$ spectrum data taken in combination with $\mathrm{B} / \mathrm{C}$ ratio data. The low energy $\mathrm{B} / \mathrm{C}$ data were used to fix the $\mathrm{LB}$ spectral parameter at $E_{b}=500 \mathrm{MeV}$, but $E_{b}=400-600 \mathrm{MeV}$ also provides good agreement with $\mathrm{B} / \mathrm{C}$ data. In this way, we have been able to obtain a model simultaneously fitting $p, \mathrm{He}, \bar{p}$, and $\mathrm{B} / \mathrm{C}$ data.

\section{ABUNDANCES IN COSMIC RAYS AND COSMIC RAY SOURCES}

The Galactic CR source elemental abundances are tuned (at a nominal reference energy of $100 \mathrm{GeV}$ ), by a least squares procedure, to the abundances measured by HEAO-3 (Engelmann et al., 1990) at 5.6, 7.5, 10.6, 16.2 GeV/n. Relative isotopic abundances at the source are taken equal to solar system abundances (Anders and Grevesse, 1989). The key point in the fitting procedure is to obtain the correct abundance of $\mathrm{B}$.

The LB elemental abundances are tuned simultaneously with spectra using the low energy part of the $\mathrm{B} / \mathrm{C}$ ratio and isotopic abundances at $200 \mathrm{MeV} / \mathrm{n}$ from ACE (Wiedenbeck et ald Abundance ratio, DR II 2001) and at $185 \mathrm{MeV} / \mathrm{n}$ from Ulysses (DuVernois and Thave 1996). For many elements ACE and Ulysses abundances differ by $10 \%$. For this reason, to the statistical errors we added 5\% systematic error. The fitting procedure is influenced by the adopted value of the modulation potential. The following pairs of modulation potentials give almost the same final result: (ACE, Ulysses) $=(400 \mathrm{MV}, 700$ MV), (450 MV, $600 \mathrm{MV}),(500 \mathrm{MV}, 500 \mathrm{MV}$ ). The data deviate from calculations in both directions, which mean that we are unlikely to introduce essential systematic error by assuming a wrong value of the modulation potential.

The diffusive reaccleration model with an LB component shows good overall agreement with data including secondary to primary ratios, spectra, and abundances. The derived Galactic CR source abundances and LB source abundances are plotted in Figure 2 relative to the solar system abundances (Grevesse and Sauval, 1998). The important result is that $\mathrm{CR}$ source and LB source abundances of

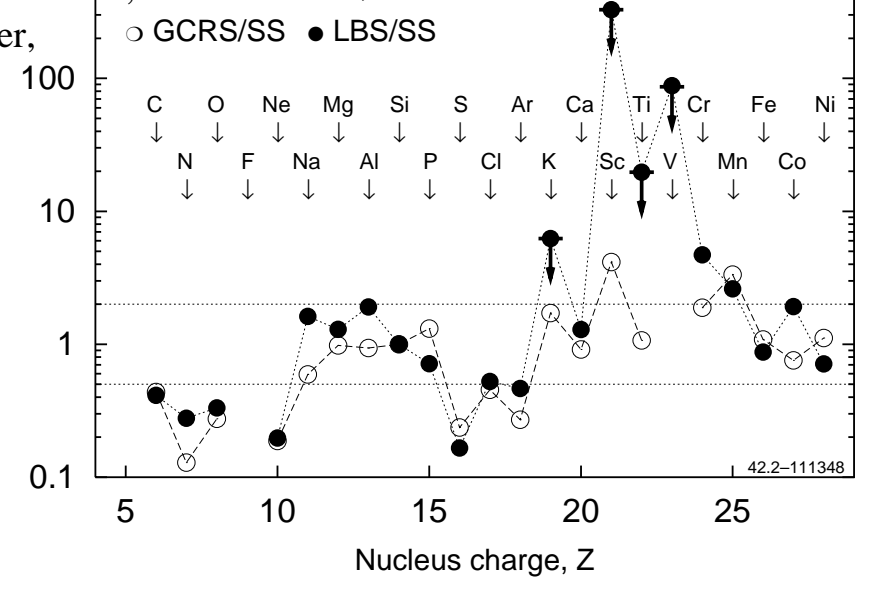

Fig. 2: Derived abundance ratios Galactic-CR-source to solar-system (GCRS/SS) and LB-source to solarsystem (LBS/SS), normalized to Si. Relative abundances for $\mathrm{K}, \mathrm{Sc}, \mathrm{Ti}, \mathrm{V}$ are shown as upper limits. The dotted lines plotted at $1 / 2$ and 2 . all major elements (C, O, Ne, Mg, $\mathrm{Si}, \mathrm{S}, \mathrm{Ar}, \mathrm{Ca}, \mathrm{Fe}, \mathrm{Ni}$ ) are in good agreement with each other. Abundances of the $\mathrm{Si}$ group, $\mathrm{Ca}, \mathrm{Fe}, \mathrm{Ni}$ are near the solar system abundances. Abundances of other elements in Galactic $\mathrm{CR}$ and LB sources are mostly consistent with each other, and with solar system abundances, within a factor of 2. Relative to silicon, C, N, $\mathrm{O}, \mathrm{Ne}, \mathrm{S}$ are underabundant both in CR source and LB. This corresponds to the well-known first-ionization-potential or volatily correlation (see, e.g., Mever et al., 1998).

Spectra of B, C, O, Fe are shown in Figure 3 for modulation levels 450 and $800 \mathrm{MV}$. In case of C, the normalization coefficient in the LB component is fixed as $a(6,12)=6.35 \times 10^{-4} \mathrm{~cm}^{-2} \mathrm{~s}^{-1} \mathrm{sr}^{-1}$ for $\eta=1$. Since the elemental abundances are tuned at both high and low energies, the calculated sub-Fe/Fe ratio also agrees well with data.

Secondary nuclei K, Sc, Ti, V appear to be overabundant in the LB sources relative to the solar system (shown as upper limits in Figure 2, though the derived absolute LB abundances are not large. The derived LB abundance of Ti does not exceed that of $\mathrm{Cr}$ while the derived abundances of $\mathrm{Sc}$ and $\mathrm{V}$ are not larger than that of $\mathrm{Mn}$. One possible reason for this excess is the uncertainty in the production cross sections, which is especially large for these 

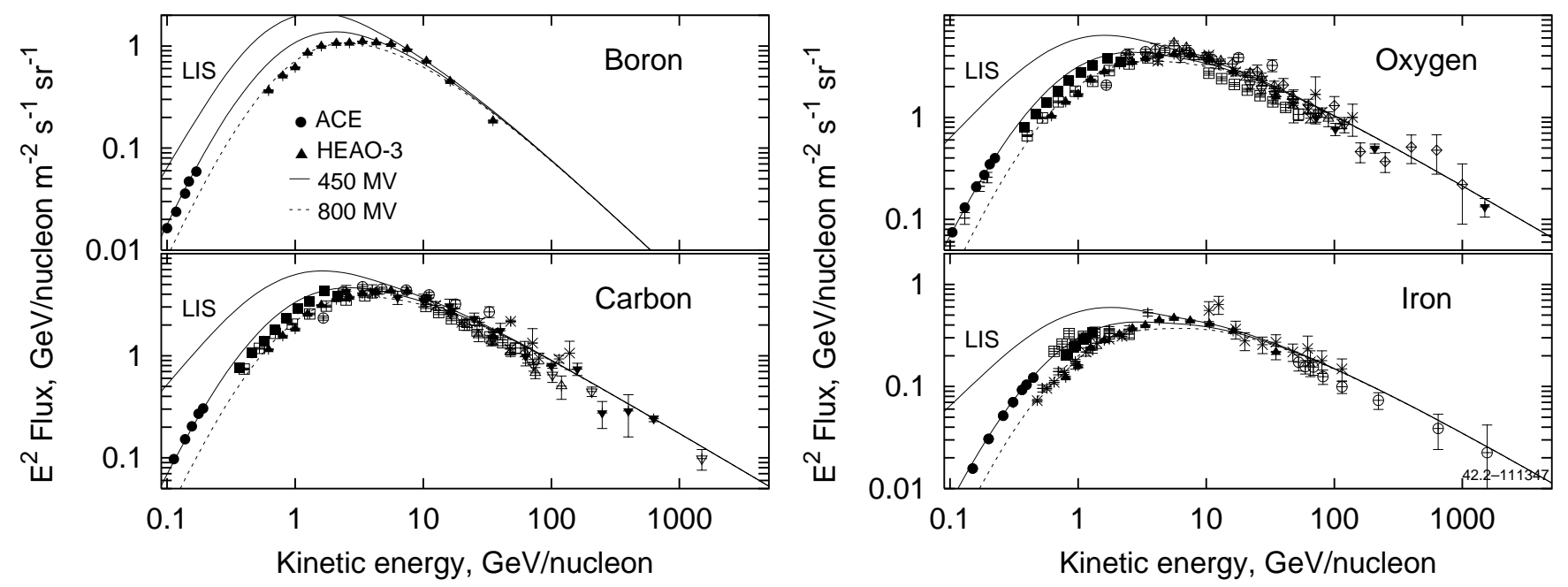

Fig. 3. Spectra of B, C, O, and Fe calculated with LB contribution. Upper curves - LIS, lower curves - modulated using force field approximation ( $\Phi=450 \mathrm{MV}$ - solid curves, $\Phi=800 \mathrm{MV}$ - dashes). Data: ACE (Davis et al., 2000, 2001), HEAO-3 (Engelmann et al., 1990), for other references see Stephens and Streitmatter (1998).

nuclei. Sometimes there is no measurement at all, while phenomenological systematics are frequently wrong by a factor of two or more. Often, there is only one measurement at $\sim 600 \mathrm{MeV} / \mathrm{n}$, which has to be extrapolated in both directions. This allows only a nearly flat Webber-type or Silberberg-Tsao-type extrapolations while the real cross sections usually have large resonances below several hundred $\mathrm{MeV}$, and decrease with energy above a few $\mathrm{GeV}$ (see, e.g., Moskalenko et al., 2001a). We note that Davis et al. (2000) used semiempirical cross sections based on Webber et al. (1990) and also predicted fluxes of sub-Fe elements which are too low.

An estimate of the overall error, which is reflected in the derived LB source abundances, can be obtained by assuming the absence of K, Sc, Ti, V in the LB source. Such a discrepancy is below 20\%, and can be removed by allowing the production cross sections to increase at low energies by $\sim 15-20 \%$, which seems plausible. Another possibility is errors in flux measurements of the rare CR species. Ulysses and ACE measurements are not always in agreement. Note that even for such an abundant nucleus as Fe, which is the main contributor to the sub-Fe group, the discrepancy exceeds $10 \%$, while the disagreement in abundance of $\mathrm{Sc}$ is $\sim 30 \%$.

The derived source overabundance of sub-Fe elements in the LB could also in principle arise from composition differences between the ISM in the LB and solar or Galactic average ISM. This is suggested by the fact that the relative abundances of secondary elements in the LB sources are systematically larger than in the Galactic CR sources (Figure 2).

\section{Isotopic distributions in cosmic rays}

Be and B isotopes are assumed all secondary, thus there is no possibility to tune them. Our calculation shows perfect agreement with the data on relative isotopic abundances of $\mathrm{Be}$ and $\mathrm{B}$. This is in contrast with a "standard" reacceleration model, where we obtained a $15 \%$ discrepancy with relative abundances of $\mathrm{Be}$ and Be isotopes (Strong and Moskalenko, 2001). Abundances of stable isotopes of other elements are not very conclusive because they are present in the sources, but $\mathrm{O}$ and $\mathrm{Si}$ isotopic distributions still agree very well with data by ACE, Ulysses, and Voyager assuming only ${ }^{16} \mathrm{O}$ and ${ }^{28} \mathrm{Si}$ isotopes are present in the $\mathrm{LB}$ component. $\mathrm{C}$ and $\mathrm{N}$ isotopic distributions do not agree too well, but this may point to a problem with cross sections. The calculated ratio ${ }^{13} \mathrm{C} /{ }^{12} \mathrm{C} \sim 0.11$ at 120 $\mathrm{MeV} / \mathrm{n}(\Phi=500 \mathrm{MV})$ in the model with LB contribution is still a factor $\sim 1.5$ too large compared to the measured value, $0.0629 \pm 0.0033$ (Voyager 50-130 MeV/n, Webber et al., 1996) and $0.078 \pm 0.011$ (Ulysses 100-200 MeV/n, DuVernois et al., 1996a), which may be connected in part with overproduction of ${ }^{13} \mathrm{C}$ by ${ }^{15} \mathrm{~N}$. If we replace the cumulative cross section ${ }^{15} \mathrm{~N}+p \rightarrow{ }^{13} \mathrm{C}$ with cross section ${ }^{14} \mathrm{~N}+p \rightarrow{ }^{13} \mathrm{C}$, the calculated ratio ${ }^{13} \mathrm{C} /{ }^{12} \mathrm{C}$ will be lowered by $10 \%$ as estimated. Assuming the absence of the isotope ${ }^{13} \mathrm{C}$ in the Galactic CR sources gives another $10 \%$ reduction. Altogether these corrections yield ${ }^{13} \mathrm{C} /{ }^{12} \mathrm{C} \sim 0.09$, close to the data.

In case of radioactive isotopes $\mathrm{Be}, \mathrm{Al}, \mathrm{Cl}$, usually used as "clocks" in $\mathrm{CR}$, the agreement with the most accurate low energy data by ACE is very good and all the ratios are consistent with each other indicating that a halo height $z_{h}=4$ 
$\mathrm{kpc}$ is a good estimate. Higher energy data by ISOMAX (Be) are also consistent with calculations considering the large error bars. The ${ }^{54} \mathrm{Mn} / \mathrm{Mn}$ ratio indicates a somewhat smaller halo, but this may be related to uncertainty in its half-life and/or production cross section. The half-life of ${ }^{54} \mathrm{Mn}$ against $\beta^{-}$decay is the most uncertain among the four radioactive isotopes - it is only one which is not measured directly. Apart from the half-life, a possible source of errors can be production cross sections of $\mathrm{Mn}$ isotopes. Only the reaction ${ }^{n a t} \mathrm{Fe}+p \rightarrow{ }^{54} \mathrm{Mn}$ on a natural sample of Fe has been measured well enough (see compilation by Mashnik et al., 1998).

\section{CONCLUSION}

In a previous paper we have shown that the $\bar{p}$ flux and $\mathrm{B} / \mathrm{C}$ ratio appear to be inconsistent with measurements when computed in standard diffusion/reaccelation models. In this paper we have demonstrated that this discrepancy can be resolved if some part of the local CR consists of a "fresh" component accelerated in the LB. The independent evidence for SN activity in the solar vicinity supports this idea.

Combining the measurements of the $\bar{p}$ flux and $\mathrm{B} / \mathrm{C}$ ratio to fix the diffusion coefficient, we have been able to construct a model consistent with measurements of important nuclei ratios in CR and derive elemental abundances in the LB. Calculated isotopic abundance distributions of $\mathrm{Be}$ and $\mathrm{B}$ are in perfect agreement with $\mathrm{CR}$ data. The abundances of three "radioactive clock" isotopes in $\mathrm{CR}{ }^{10} \mathrm{Be},{ }^{26} \mathrm{Al},{ }^{36} \mathrm{Cl}$ are all consistent and indicate a halo size $z_{h} \sim 4 \mathrm{kpc}$ based on the ACE data. ${ }^{54} \mathrm{Mn}$ indicated a smaller halo, but this may be related to its half-life uncertainty and/or cross section errors. The derived fraction of LB component in CR is small compared to Galactic CR and has a steep spectrum with a cutoff above several hundred $\mathrm{MeV} / \mathrm{n}$. Other experimental data (except maybe the overabundance of $\mathrm{Sc}, \mathrm{Ti}, \mathrm{V}$ ) do not contradict this hypothesis. The derived source overabundance of sub-Fe elements in the LB may be caused by trivial uncertainties in the production cross sections, or could in principle arise from composition and/or evolution differences between the ISM in the LB and solar or Galactic average ISM.

Recently there has appeared some indication (Huang et al., 2001) that the atmospheric contribution to the $\bar{p}$ flux measured in the upper atmosphere is underestimated by $\sim 30 \%$. This means that the flux of $\bar{p}$ 's in CR in reality may be lower at the top of the atmosphere by at least 25-30\%. If the latter is true, the reacceleration model (even without LB) could still be the best one to describe propagation of nucleon species in the Galaxy.

\section{ACKNOWLEDGEMENTS}

The authors are grateful to M. Wiedenbeck for providing the ACE isotopic abundances. I. V. M. is grateful to the Gamma Ray Group of the MPI für extraterrestrische Physik, where a part of this work has been done, for hospitality. I. V. M. and S. G. M. acknowledge partial support from a NASA ATP grant.

\section{REFERENCES}

Alcaraz, J., et al., Cosmic protons, Phys. Lett. B, 490, 27-35, 2000.

Anders, E., and N. Grevesse, Abundances of the elements - meteoritic and solar, Geochim. Cosmochim. Acta, 53, 197-214, 1989.

Asaoka, Y., et al., Measurements of cosmic-ray low-energy antiproton and proton spectra in a transient period of solar field reversal, Phys. Rev. Lett., 88, 051101, 2002.

Basini, G., et al., The Flux of Cosmic Ray Antiprotons from 3.7 to $24 \mathrm{GeV}$, in Proc. 26th Int. Cosmic Ray Conf. (Salt Lake City), 3, 77-80, 1999.

Berghöfer, T. W., and D. Breitschwerdt, The origin of the young stellar population in the solar neighborhood - a link to the formation of the Local Bubble?, Astron. Astrophys., 390, 299-306, 2002.

Boezio, M., et al., The cosmic-ray antiproton flux between 3 and $49 \mathrm{GeV}$, Astrophys. J., 561, 787-799, 2001.

Bykov, A. M., and G. D. Fleishman, On non-thermal particle generation in superbubbles, Mon. Not. Royal Astron. Soc., 255, 269-275, 1992.

Davis, A. J., et al., On the low energy decrease in Galactic cosmic ray secondary/primary ratios, in Acceleration and Transport of Energetic Particles Observed in the Heliosphere (ACE-2000), edited by R. A. Mewaldt et al., AIP Conf. Proc., 528, pp.421-424, AIP, New York, 2000.

Davis, A. J., et al., The evolution of Galactic cosmic ray element spectra from solar minimum to solar maximum, in Proc. 27th Int. Cosmic Ray Conf. (Hamburg), 3971-3974, 2001.

DuVernois, M. A., and M. R. Thayer, The elemental composition of the Galactic cosmic-ray source: ULYSSES high-energy telescope results, Astrophys. J., 465, 982-984, 1996.

DuVernois, M. A., M. Garcia-Munoz, K. R. Pyle, et al., The isotopic composition of Galactic cosmic-ray elements 
from carbon to silicon: the combined release and radiation effects satellite investigation, Astrophys. J., 466, 457-472, 1996a.

DuVernois, M. A., J. A. Simpson, and M. R. Thayer, Interstellar propagation of cosmic rays: analysis of the ULYSSES primary and secondary elemental abundances, Astron. Astrophys., 316, 555-563, $1996 \mathrm{~b}$.

Engelmann, J. J., et al., Charge composition and energy spectra of cosmic-ray nuclei for elements from Be to NI Results from HEAO-3-C2, Astron. Astrophys., 233, 96-111, 1990.

Grevesse, N., and A. J. Sauval, Standard solar composition, Space Sci. Rev., 85, 161-174, 1998.

Huang, C. Y., L. Derome, and M. Buénerd, Secondary antiprotons from Cosmic Ray interactions in the Atmosphere, in Proc. 27th Int. Cosmic Ray Conf. (Hamburg), 1707-1710, 2001.

Jones, F. C., A. Lukasiak, V. Ptuskin, et al., The modified weighted slab technique: models and results, Astrophys. J., 547, 264-271, 2001.

Knie, K., G. Korschinek, T. Faestermann, et al., Indication for supernova produced ${ }^{60} \mathrm{Fe}$ activity on Earth, Phys. Rev. Lett., 83, 18-21, 1999.

Lukasiak, A., F. B. McDonald, and W. R. Webber, Voyager measurements of the charge and isotopic composition of cosmic ray $\mathrm{Li}, \mathrm{Be}$ and $\mathrm{B}$ nuclei and implications for their production in the Galaxy, in Proc. 26th Int. Cosmic Ray Conf. (Salt Lake City), 3, 41-44, 1999.

Maíz-Apellániz, J., The origin of the Local Bubble, Astrophys. J., 560, L83-L86, 2001.

Mashnik, S. G., A. J. Sierk, K. A. Van Riper, et al., Production and validation of isotope production cross section libraries for neutrons and protons to $1.7 \mathrm{GeV}$, in Proc. 4th Workshop on Simulating Accelerator Radiation Environments, edited by T.A. Gabriel, pp.151-161, ORNL, Oak Ridge, 1998. (nucl-th/9812071)

Meyer, J.-P., L. O'C. Drury, and D. C. Ellison, A cosmic-ray composition controlled by volatility and a/q ratio. SNR shock acceleration of gas and dust, Spa. Sci. Rev., 86, 179-201, 1998.

Molnar, A., and M. Simon, A new calculation of the interstellar secondary cosmic ray, in Proc. 27th Int. Cosmic Ray Conf. (Hamburg), 1877-1879, 2001.

Moskalenko, I. V., S. G. Mashnik, and A. W. Strong, New calculation of radioactive secondaries in cosmic rays, in Proc. 27th Int. Cosmic Ray Conf. (Hamburg), 1836-1839, 2001 a.

Moskalenko, I. V., A. W. Strong, J. F. Ormes, et al., Secondary antiprotons in cosmic rays, in Proc. 27th Int. Cosmic Ray Conf. (Hamburg), 1868-1871, 2001b.

Moskalenko, I. V., A. W. Strong, J. F. Ormes, et al., Secondary antiprotons and propagation of cosmic rays in the Galaxy and heliosphere, Astrophys. J., 565, 280-296, 2002.

Moskalenko, I. V., A. W. Strong, S. G. Mashnik, et al., Challenging cosmic ray propagation with antiprotons. Evidence for a "fresh" nuclei component?, Astrophys. J., 586, in press, 2003. (astro-ph/0210480)

Orito, S., et al., Precision measurement of cosmic-ray antiproton spectrum, Phys. Rev. Lett., 84, 1078-1081, 2000.

Sanuki, T., et al., Precise measurement of cosmic-ray proton and helium spectra with the BESS spectrometer, Astrophys. J., 545, 1135-1142, 2000.

Sfeir, D. M., R. Lallement, F. Crifo, et al., Mapping the contours of the Local bubble: preliminary results, Astron. Astrophys., 346, 785-797, 1999.

Sonett, C. P., G. E. Morfill, and J. R. Jokipii, Interstellar shock-waves and Be-10 from ice cores, Nature, 330, 458-460, 1987.

Stephens, S. A., and R. A. Streitmatter, Cosmic-ray propagation in the Galaxy: techniques and the mean matter traversal, Astrophys. J., 505, 266-277, 1998.

Strong, A. W., and I. V. Moskalenko, Propagation of cosmic-ray nucleons in the Galaxy, Astrophys. J., 509, 212-228, 1998.

Strong, A. W., and I. V. Moskalenko, Models for Galactic cosmic-ray propagation, Adv. Space Res., 27, 717-726, 2001.

Webber, W. R., J. C. Kish, and D. A. Schrier, Formula for calculating partial cross-sections for nuclear-reactions of nuclei with $E \geq 200 \mathrm{MeV} /$ nucleon in hydrogen targets, Phys. Rev. C, 41, 566-571, 1990.

Webber, W. R., A. Lukasiak, F. B. McDonald, et al., New high-statistical - high-resolution measurements of the cosmic-ray CNO isotopes from a 17 year study using the Voyager 1 and 2 spacecraft, Astrophys. J., 457, 435439, 1996.

Wiedenbeck, M. E., et al., The origin of primary cosmic rays: constraints from ACE elemental and isotopic composition observations, Spa. Sci. Rev., 99, 15-26, 2001.

\section{E-mail address of I.V. Moskalenko: imos@milkyway.gsfc.nasa.gov}

